\title{
Postoperative Imaging Findings following Sigmoid Sinus Wall Reconstruction for Pulse Synchronous Tinnitus
}

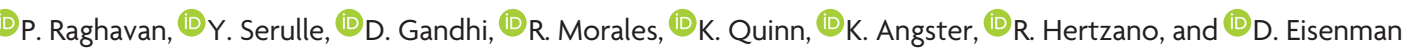

\begin{abstract}
BACKGROUND AND PURPOSE: Transmastoid sigmoid sinus wall reconstruction is a surgical technique increasingly used for the treatment of pulsatile tinnitus arising from sigmoid sinus wall anomalies. The imaging appearance of the temporal bone following this procedure has not been well-characterized. The purpose of this study was to evaluate the postoperative imaging appearance in a group of patients who underwent this procedure.
\end{abstract}

MATERIALS AND METHODS: The medical records of 40 consecutive patients who underwent transmastoid sigmoid sinus wall reconstruction were reviewed. Thirteen of 40 patients underwent postoperative imaging. Nineteen CT and 7 MR imaging examinations were assessed for the characteristics of the materials used for reconstruction, the impact of these on the adjacent sigmoid sinus, and complications.

RESULTS: Tinnitus resolved in 38 of 40 patients. Nine patients were imaged postoperatively for suspected complications, including dural sinus thrombosis, facial swelling, and wound drainage. Two patients underwent imaging for persistent tinnitus, and 2, for development of tinnitus on the side contralateral to the side of surgery. The materials used for reconstruction (NeuroAlloderm, HydroSet, bone pate) demonstrated characteristic imaging appearances and could be consistently identified. In 5 of 13 patients, there was extrinsic compression of the sigmoid sinus by graft material. Dural sinus thrombosis occurred in 2 patients.

CONCLUSIONS: The imaging findings following sigmoid sinus wall repair are characteristic. Graft materials may result in extrinsic compression of the sigmoid sinus, and this finding may be confused with dural venous thrombosis. Awareness of the imaging characteristics of the graft materials used enables this differentiation.

ABBREVIATIONS: PST = pulse synchronous tinnitus; $S$ SWR $=$ sigmoid sinus wall reconstruction

T innitus may be categorized as subjective, when it originates in either the peripheral or central auditory system and is perceived only by the patient, or objective, when it arises from a mechanical somatosound. ${ }^{1}$ Pulsatile, or pulse synchronous, tinnitus (PST) usually arises from the abnormal self-perception of one's vascular flow. PST is a potentially disabling symptom, which may profoundly impact daily functioning. ${ }^{2}$ Although PST can arise from a number of venous and arterial abnormalities, ${ }^{2,3}$ venous PST accounts for most cases encountered in clinical practice. ${ }^{4}$

It is increasingly recognized that sigmoid sinus wall anomalies, which include thinning and dehiscence of the sigmoid sinus plate

Received May 3, 2015; accepted after revision June 9.

From the Departments of Radiology (P.R., Y.S., D.G., R.M.) and Otolaryngology (K.Q., K.A., R.H., D.E.), University of Maryland Medical Center, Baltimore, Maryland.

Prashant Raghavan and Yafell Serulle shared equally in the authorship of this article and are co-first authors.

Please address correspondence to Prashant Raghavan, MBBS, Department of Radiology, University of Maryland Medical Center, 22 S Greene St, Baltimore, MD 21201; e-mail: Prashant.raghavan@gmail.com

http://dx.doi.org/10.3174/ajnr.A4511 with or without an associated diverticulum, are a frequently encountered and surgically correctable cause of PST. ${ }^{5-7}$ Both open surgical and endovascular interventions have proved successful in the amelioration of PST in such patients. ${ }^{8-10}$ Sigmoid sinus wall reconstruction (SSWR) is increasingly being performed via an extraluminal, transmastoid approach. ${ }^{11}$ The goal of the procedure is to bridge the bony dehiscence and reconstruct the wall of the sinus, thereby eliminating audible turbulence and interrupting the transmission of mural vibrations via the mastoid air cells. The purpose of this study was to describe the imaging findings in patients who have undergone SSWR and to evaluate the imaging characteristics of complications arising from this procedure.

\section{MATERIALS AND METHODS \\ Patients}

This retrospective, anonymized, single-center study was performed in accordance with the Health Insurance Portability and Accountability Act. Requirement for informed consent was waived by the institutional review board. The medical records of 40 consecutive patients ( 35 females; median age, 38 years; range, 
14-70 years) who underwent sigmoid sinus repair between May 2007 and January 2015 were reviewed. Patients were selected for the procedure after comprehensive clinical, audiometric, and tympanometric evaluations. All patients had undergone preoperative CT imaging examinations demonstrating the presence of sigmoid sinus wall anomalies (Fig 1). Postoperative imaging was obtained in 13 of the 40 patients, either for persistent or recurrent PST following surgery (4/40) or when there was concern for surgical complications $(9 / 40)$.

\section{Surgical Technique}

The standardized technique of SSWR has been previously described (Fig 2). ${ }^{5}$ Briefly, an extended mastoidectomy is performed, followed by skeletonization of the sigmoid sinus. The affected area is then decompressed to a normal-appearing sinus wall. The surrounding sinus wall and dura are undermined away from the posterior petrous face. Ectatic portions of the sinus are reduced with bipolar cautery. A soft-tissue graft of the temporalis fascia or AlloDerm (LifeCell, Branchburg, New Jersey), an acellular collagen matrix, is appropriately sized and inserted between the dura and posterior fossa bony plate, reconstructing the softtissue sinus wall. The graft is held in place without suturing by the intracranial and intravascular pressure. For hemostasis, especially if there is violation of the diverticulum intraoperatively, Surgicel (Ethicon, Somerville, New Jersey) is sometimes inserted deep into
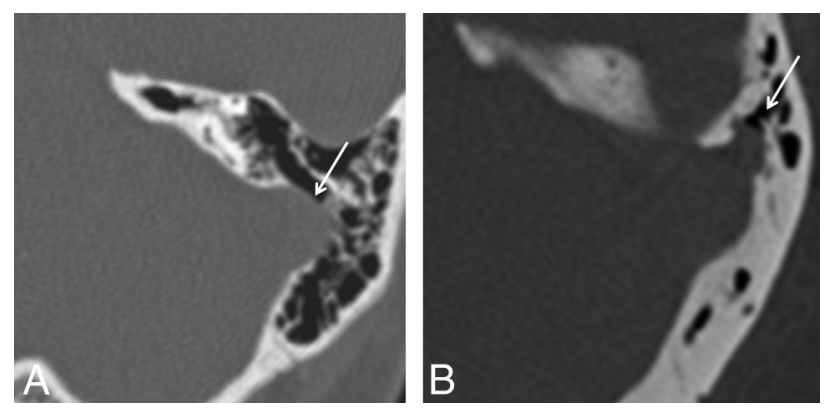

FIG 1. CT features of sigmoid wall anomalies. A, Axial CT image of the temporal bone demonstrates dehiscence of the left sigmoid sinus wall (arrow). B, Axial CT image of the temporal bone in a different patient demonstrates a small left sigmoid sinus diverticulum (arrow). Both patients presented with left pulse synchronous tinnitus. the soft-tissue graft. The bony defect is reconstructed with HydroSet Injectable HA Bone Substitute (Stryker, Kalamazoo, Michigan), a calcium phosphate cement that converts to hydroxyapatite. Autologous bone pate, derived from bone dust produced by drilling of the mastoid temporal bone that mixes with blood oozing in the surgical field to form a jellylike layer, is added for additional reinforcement, external to the HydroSet. The rationale behind reinforcing the soft-tissue reconstruction with the addition of a rigid layer (HydroSet and bone pate) is to diminish transmission of vibration of the exposed venous wall. ${ }^{5}$ In summary, the reconstruction comprises 3 layers: soft-tissue graft, HydroSet, and bone pate, from innermost to outermost.

\section{Imaging Studies}

Nineteen postoperative CT examinations were reviewed. Seven patients had a single CT, and 6 patients had 2 CT examinations following surgery. Iodinated intravenous contrast was administered in 8 patients. Seven MR imaging examinations were performed in 4 patients following surgery ( 1 nonenhanced MR imaging in conjunction with a $2 \mathrm{D}$ time-of-flight MR venogram and 6 gadolinium-enhanced MR imaging examinations, 3 of which included contrast-enhanced MR venography). Preoperative temporal bone CTs were available in all patients for comparison.

Scanning parameters for temporal bone CT examinations were as follows: section thickness, $0.67 \mathrm{~mm}$ at a 0.33 -mm interval, $140 \mathrm{kV}$ (peak), $350 \mathrm{mAs}$ at $64 \times 0.625 \mathrm{~mm}$ collimation, a 0.358 pitch, rotation time of 0.5 , and FOV of $200 \mathrm{~mm}$. Images were reconstructed in axial and coronal planes. Intravenous contrast (100 mL of iohexol injection, Omnipaque; GE Healthcare, Piscataway, New Jersey) was administered at $1.5 \mathrm{~mL} / \mathrm{s}$ followed by a 50 -mL saline flush, with automatic image acquisition 20 seconds after the appearance of contrast in the common carotid arteries. MR images were obtained by using a 1.5T (Avanto; Siemens, Erlangen, Germany) MR imaging scanner. Protocols included standard T1, T2, FLAIR, and susceptibility- and diffusion-weighted images of the brain and 2D time-of-flight venography.
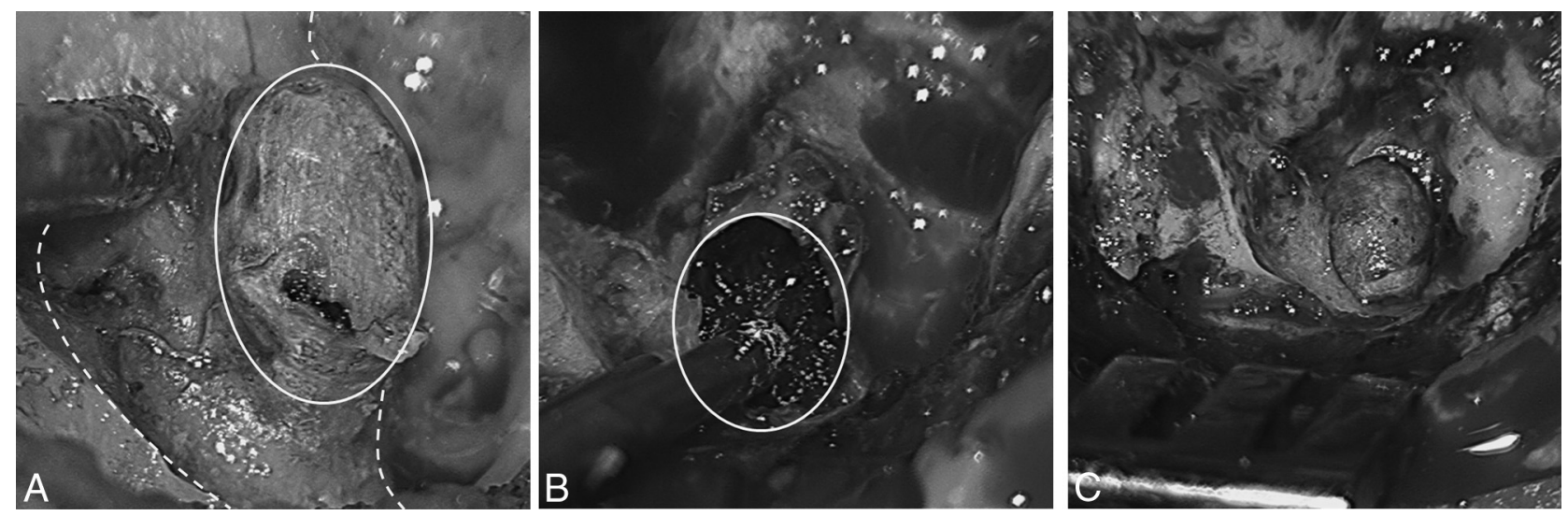

FIG 2. Intraoperative findings in sigmoid sinus wall repair. A, Appearance of a right sigmoid sinus diverticulum during transmastoid surgery. Dashed lines show the outline of a normal sigmoid sinus; the oval indicates a diverticulum. $B$, Postreduction of diverticulum. $C$, Postrepair of a sinus wall. Note the soft-tissue graft reinforcing the wall of the sigmoid sinus. Following this step, the bone defect is repaired with synthetic bone cement (HydroSet) and autologous bone pate. In all images, the left of the figure corresponds to the back of the patient (posterior head). 
Summary of patient demographics and clinical, imaging, and surgical features

\begin{tabular}{|c|c|c|c|c|c|c|c|c|}
\hline No. & $\begin{array}{l}\text { Age } \\
\text { (yr) }\end{array}$ & Sex & Side & $\begin{array}{l}\text { Indication } \\
\text { for SSWR }\end{array}$ & $\begin{array}{c}\text { Indication for } \\
\text { Postoperative Imaging }\end{array}$ & $\begin{array}{l}\text { Mass Effect } \\
\text { on SS }\end{array}$ & $\begin{array}{l}\text { Dural Venous } \\
\text { Sinus Thrombus }\end{array}$ & $\begin{array}{l}\text { Other } \\
\text { Findings }\end{array}$ \\
\hline 1 & 59 & $\mathrm{~F}$ & $\mathrm{~L}$ & Dehiscence & Persistent tinnitus & - & - & Dehiscent left jugular bulb \\
\hline 2 & 57 & $\mathrm{~F}$ & L & Dehiscence & Persistent tinnitus & $+^{a}$ & - & \\
\hline 3 & 41 & $\mathrm{~F}$ & $\mathrm{R}$ & $\begin{array}{l}\text { Dehiscence }+ \\
\text { encephalocele }\end{array}$ & Leak from wound & - & - & $\begin{array}{l}\text { Tegmen tympani } \\
\text { encephalocele }+ \text { fluid collection }\end{array}$ \\
\hline 4 & 62 & $\mathrm{~F}$ & L & Dehiscence & Headache + visual changes & - & - & \\
\hline 5 & 31 & $\mathrm{~F}$ & $R>L$ & Diverticulum & L tinnitus & - & - & $L$ dehiscence \\
\hline 6 & 37 & $\mathrm{~F}$ & L & Dehiscence & Headache + neck pain & + & - & \\
\hline 7 & 38 & $\mathrm{~F}$ & $\mathrm{R}$ & Diverticulum & Headache & - & + & \\
\hline 8 & 14 & M & $\mathrm{R}$ & Dehiscence & $\mathrm{L}$ tinnitus & - & - & $\mathrm{L}$ dehiscence \\
\hline 9 & 21 & $\mathrm{~F}$ & $\mathrm{R}$ & Dehiscence & Headache + visual changes & - & - & \\
\hline 10 & 44 & M & $\mathrm{R}$ & Diverticulum & Headache & - & - & \\
\hline 11 & 20 & $\mathrm{~F}$ & L & Dehiscence & Headache & $+^{a}$ & - & \\
\hline 12 & 46 & $\mathrm{~F}$ & $\mathrm{R}$ & Diverticulum & $\begin{array}{l}\text { Intraoperative diverticulum } \\
\text { rupture }\end{array}$ & + & - & \\
\hline 13 & 65 & $\mathrm{~F}$ & $\mathrm{R}$ & Diverticulum & Right facial swelling & + & + & \\
\hline
\end{tabular}

Note:-SS indicates sigmoid sinus; R, right; L, left.

${ }^{a} \mathrm{C} T$ was initially interpreted as dural sinus thrombosis.

\section{Image Analysis}

A qualitative examination was performed by 2 neuroradiologists, with 10 and 15 years of experience, blinded to clinical data, who reviewed all images individually. In case of disagreement, the radiologists reviewed the images together on the same workstation, to reach a consensus. All findings pertaining to graft materials were confirmed by the operating surgeon.

Preoperative temporal bone CTs were evaluated for the presence of diverticula or dehiscences. The maximal transverse dimensions of the diverticula and the maximal transverse width of the dehiscences were documented. The postoperative images were evaluated for the presence and appearance (thickness, attenuation) of the surgical material used. These included the softtissue materials (temporalis fascia graft, AlloDerm, and Surgicel), HydroSet, and bone pate. Density was measured by placing ROIs at the center of each type of graft material (soft tissue, HydroSet, bone pate), ensuring that adjacent structures were not inadvertently included. The maximal transverse dimension of the surgical soft-tissue materials was measured in the axial plane. The presence of mass effect on the sigmoid sinus by the soft-tissue graft was also determined. Mass effect was defined when there was direct apposition of the soft-tissue material on the sigmoid sinus, resulting in $>50 \%$ reduction of the caliber of the sinus lumen in the transverse plane. Also evaluated were the presence of dural venous sinus thrombosis, fluid collections at the operative site, and any other findings that explained symptomatology.

\section{RESULTS}

Forty patients underwent SSWR for PST (5 male, 35 female; 14-70 years of age; median age, 38 years). There were 24 patients with dehiscence and 16 with diverticula. Average dehiscence width was $5 \mathrm{~mm}$ (range, 2-9 $\mathrm{mm}$ ), and the average maximal diverticulum size was $6 \mathrm{~mm}$ (range, 3-10 mm). The standardized surgical procedure (see "Materials and Methods") was used in all patients. Resolution of PST immediately following surgery occurred in 38/40 patients. Postoperative imaging was performed in those patients who had persistent PST following surgery $(n=2)$, late recurrence (or new occurrence) of tinnitus on the contralateral side $(n=2)$ within 2 years of surgery, or in those cases in which there was concern for surgical complications $(n=9)$. These indications included headache with or without visual disturbances $(n=6)$, concern for dural thrombus from intraoperative diverticulum rupture $(n=1)$, fluid collection at the operative site $(n=1)$, and facial swelling and erythema $(n=1)$.

Of the 13 patients with postoperative imaging, 2 were male and 11 were female, with ages ranging from 14 to 65 years (median age, 41 years) (Table). The time interval between operative intervention and first postoperative examination ranged from 1 day to 2 years (median interval, 14 days). Seven patients presented with right PST; 5, with left PST; and 1 had bilateral PST with rightsided dominance. Imaging findings on preoperative CT scans included sigmoid sinus dehiscence $(n=8)$ and sigmoid sinus diverticulum $(n=5)$. Average dehiscence width was $4 \mathrm{~mm}$ (range, $2-7$ $\mathrm{mm}$ ), and average maximal diverticulum size was $5 \mathrm{~mm}$ (range, 2-10 $\mathrm{mm})$.

In all 13 patients, SSWR was performed with soft-tissue and rigid reinforcement as previously described. In 6 patients, reconstruction was performed with AlloDerm; in 6, with temporalis fascia graft; and in 1 patient, both AlloDerm and temporalis fascia were used. Surgicel was used for hemostasis, especially when there was violation of the diverticulum during surgical reduction. In all patients, HydroSet was used to reconstruct the osseous defect, and in 11 patients, autologous bone pate was used for additional reinforcement. One patient underwent simultaneous mesh reconstruction of a tegmen tympani meningocele.

\section{Imaging Findings}

The 3 layers used in SSWR were consistently identified in all patients on imaging. Soft-tissue material between the sigmoid sinus and the sigmoid plate, comprising AlloDerm and/or temporalis fascia graft with or without Surgicel, was identified in all postoperative scans (Fig $3 A$ ) as crescentic, extraluminal, low-attenuation material relative to the enhanced sigmoid sinus (approximately $30-60 \mathrm{HU}$ [Hounsfield units]). It was not possible to differentiate the individual soft-tissue components on the basis of CT imaging characteristics. Although the thickness of the temporalis fascia and AlloDerm used in surgery is only between 1 and $2 \mathrm{~mm}$, the average thickness of the soft-tissue material on CT was $5.1 \mathrm{~mm}$ 
(range, 2-8 $\mathrm{mm}$ ). This difference may be attributed to a number of factors including variability of blood absorption by the Surgicel when used, granulation tissue, and focal hemorrhage.

HydroSet was identified as sharply demarcated hyperattenuated material (1400-1500 HU) conforming to the size and shape of dehiscence (Fig 3B). Bone pate was identified as amorphous ill-defined hyperattenuation ( $400-500 \mathrm{HU})$ placed external to the HydroSet (Fig 3B).

The soft-tissue graft demonstrated intermediate-to-high signal on T1- and T2-weighted MR imaging (Fig 4) and intermediate signal on gradient-echo sequences (Fig 5D). On gadolinium-enhanced images, no enhancement was observed in the immediate postoperative period. Four weeks following surgery, however, enhancement was present, presumably due to the formation of granulation tissue (Fig 4B, -C).

The degree of mass effect exerted by the soft-tissue material on the adjacent sigmoid sinus was variable. In $5 / 13$ patients, there was narrowing of the lumen $(>50 \%$ decrease in caliber compared with preoperative images) (Fig 5). Two of these patients presented with headaches; 1 , with persistent PST; and 1, with unrelated facial cellulitis (see below). In 1 patient who was imaged due to intraoperative diverticulum violation, the mass effect resulted in no symptoms.
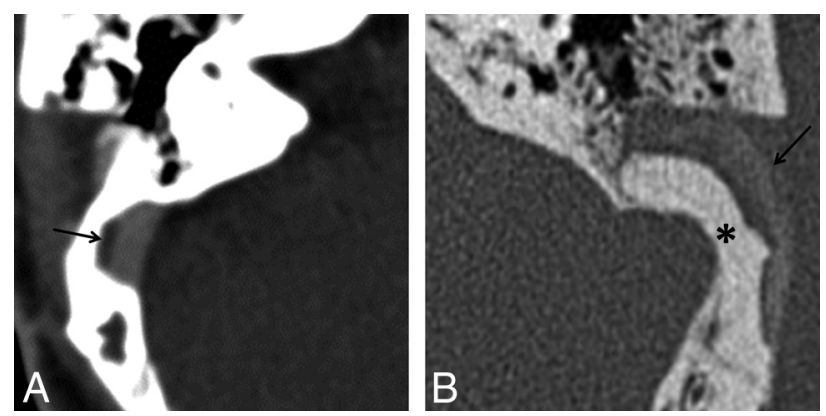

FIG 3. Postoperative $C T$ imaging. Axial $C T$ image of the temporal bone in a soft-tissue window $(A)$ demonstrates relatively hypoattenuated material (arrow) lateral to the contrast-enhanced sigmoid sinus representing soft tissue graft. $B$, On bone window (different patient), HydroSet is identified as sharply demarcated attenuated material (asterisk) conforming to the size and shape of the dehiscence. Bone pate is identified lateral to the HydroSet as amorphous ill-defined hyperattenuation (arrow).
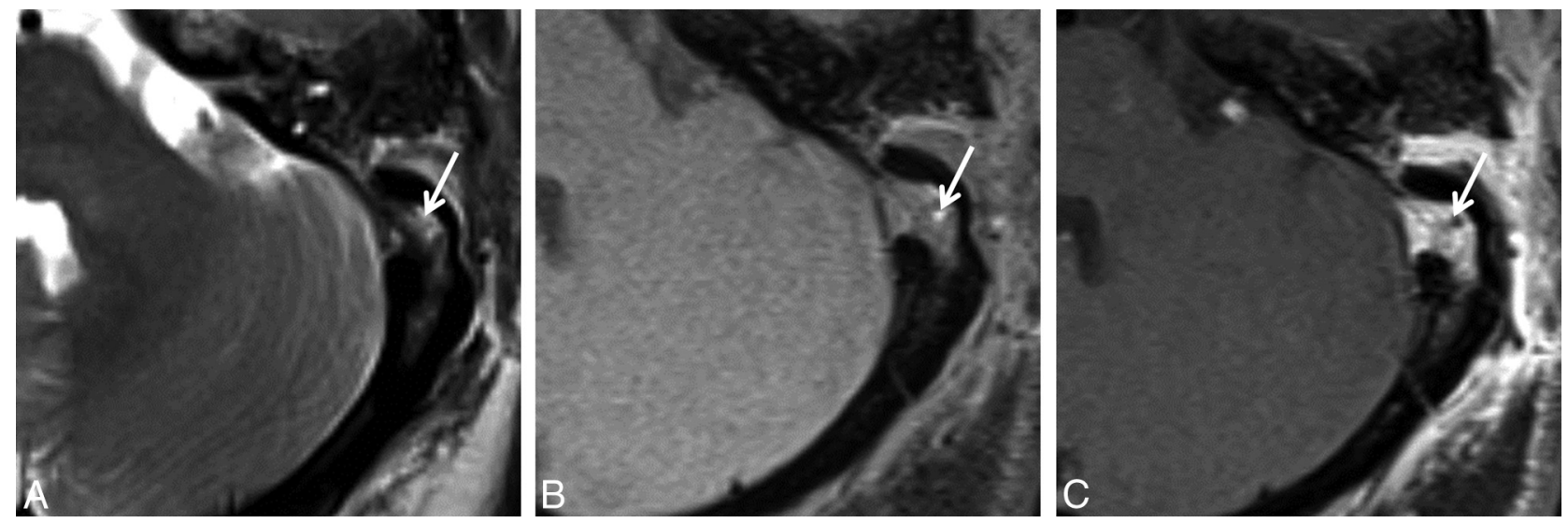

FIG 4. Postoperative MR imaging. Axial T2- $(A)$, precontrast T1- $(B)$, and postcontrast T1-weighted $(C)$ images demonstrate a temporalis fascia graft (arrow) between the sigmoid sinus and the reconstructed sigmoid sinus wall. The graft demonstrates intermediate-to-high signal on both T1- and T2-weighted images and contrast enhancement. These images were obtained 28 days following surgery. 

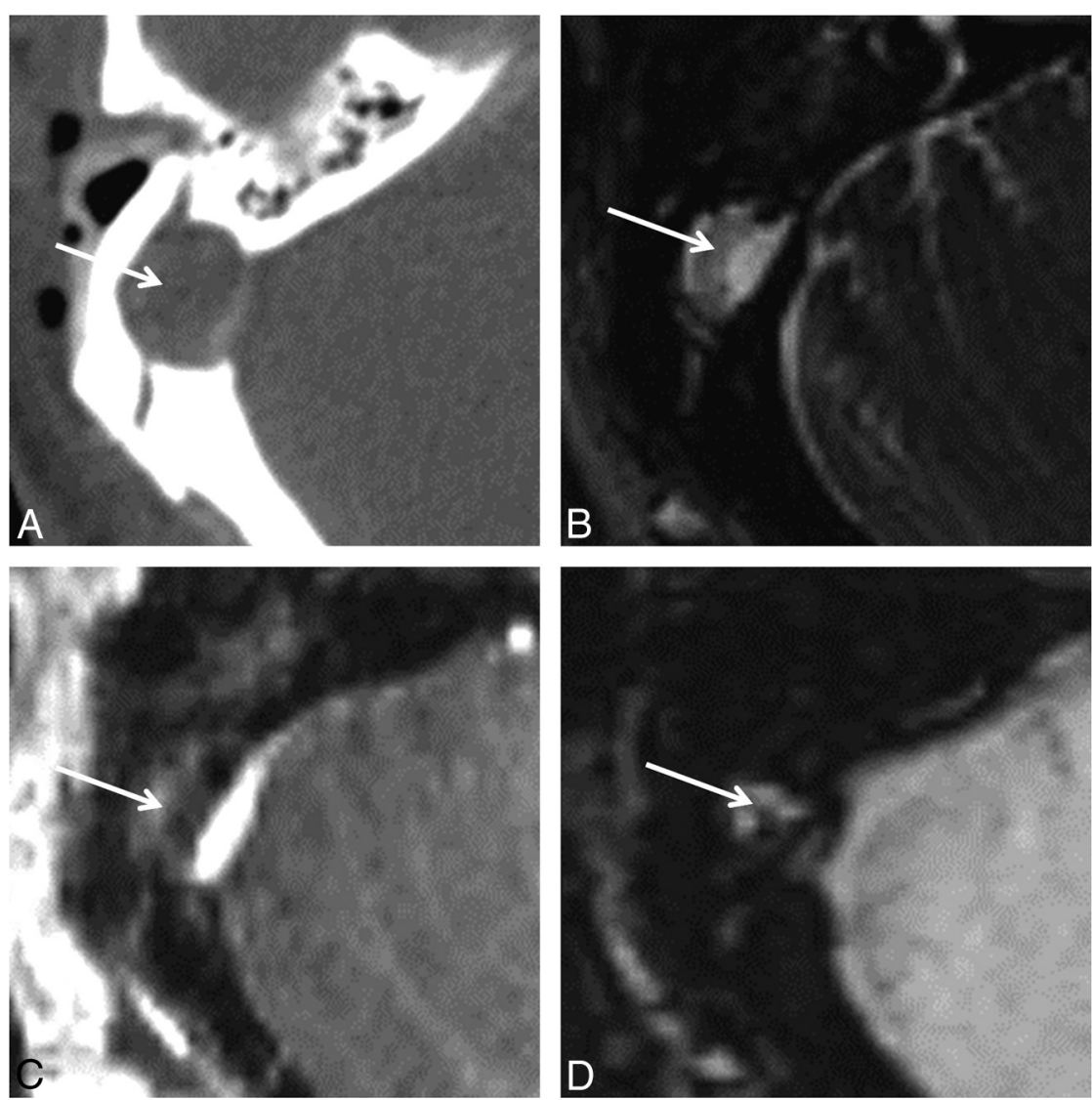

FIG 5. Mass effect on the sigmoid sinus following sigmoid sinus wall repair. A 46-year-old woman status post right diverticulum repair. Images were obtained 1 day following surgery. Postcontrast axial CT image $(A)$ demonstrates significant mass effect caused by the soft-tissue graft (arrow), resulting in severe narrowing of the sigmoid sinus. Axial T2 $(B)$ and postcontrast T1-weighted $(C)$ images. The graft is intermediate-to-high signal on T2-weighted images. Note the lack of enhancement of the graft in the immediate postoperative period. The soft-tissue material, unlike thrombus, is not hypointense on the susceptibility-weighted image $(D)$.

abnormalities historically has been endovascular embolization, ${ }^{13}$ more recently, the open transmastoid approach has become the norm. ${ }^{5,14,15}$ Otto et al $^{14}$ described 5 patients with unilateral or bilateral PST with sigmoid sinus wall abnormalities on imaging, 3 of whom underwent surgical intervention with resurfacing of the sinus with resolution of symptoms. More recently, Harvey et al $^{11}$ reported a series of 33 patients with sigmoid sinus wall abnormalities who underwent treatment by using the transmastoid approach, 30 of whom responded favorably to the procedure.

The expected imaging appearance of the temporal bone following sigmoid sinus reconstruction has not been previously described, to our knowledge. Given that postoperative imaging is not routinely performed and is only indicated to rule out postsurgical complications, it behooves the radiologist to become familiar with the appearance of the various materials used to repair and reinforce the sigmoid sinus wall to distinguish normal findings from potential disease. Our observations indicate that the 3 layers used in SSWR (soft-tissue graft, HydroSet, and bone pate) are consistently recognizable and demonstrate characteristic imaging appearances.

Recovery from transmastoid SSWR is generally uneventful. In our study, the most common symptom that warranted im- aging following surgery was headache with or without visual disturbances. Thrombosis of the sigmoid/transverse sinus is the most worrisome complication of this procedure. Manipulation of the sigmoid sinus wall entails a risk for thrombosis, and sigmoid sinus thrombosis is not infrequently encountered following translabyrinthine and suboccipital craniectomy. ${ }^{16}$ Although the surgical technique used in this study does not require significant exposure or retraction of the sigmoid sinus, the risk for thrombosis does exist. To avoid this, all patients at our institution are placed on an anticoagulation regimen comprising aspirin preoperatively and aspirin and clopidogrel postoperatively. Symptomatic postoperative thrombosis was only encountered in 1 patient, and that patient was noncompliant with the regimen.

Mass effect on the sigmoid sinus from extraluminal soft-tissue material at the operative site was commonly seen in our study and is a finding that may be confused with thrombosis. Five patients of the 13 evaluated in this series had this finding, 3 of whom had headaches. Patients with mass effect were closely monitored and reported resolution of their headaches with conservative management. The mass effect likely arises from a combination of soft-tissue material (AlloDerm/temporalis fascia); Surgicel, which likely undergoes expansion in the operative bed; and a localized hematoma. Differentiation of these individual components is not possible on imaging. However, it is important to distinguish this extraluminal process (which requires close clinical observation, but is self-limiting) from intraluminal thrombosis (which may require prompt anticoagulation). A combination of $\mathrm{CT}$ and MR imaging characteristics may be useful in this regard (Figs 5 and 6). Acute thrombus is hyperattenuated on noncontrast CT, whereas extraluminal soft-tissue material typically appears as relatively hypoattenuating material and is focal, being confined to the site of repair. On MR imaging, on gradient-echo or susceptibility-weighted images, thrombus, unlike graft material, is markedly hypointense and may propagate along the course of the transverse/sigmoid sinus. If there are equivocal findings, a combination of both CT and MR imaging may be used to distinguish intraluminal thrombus from extraluminal surgical material.

Two of the 13 patients reported persistent tinnitus after surgery. In 1 patient, this was attributed to a coexistent dehiscence of the ipsilateral jugular bulb. In the second patient, no additional findings were identified that could explain persistent symptomatology. In both patients, the postoperative scans indicated that the dehiscent sigmoid sinus had been fully reconstructed. In addition, in 2 patients, PST resolved in the immediate postoperative 

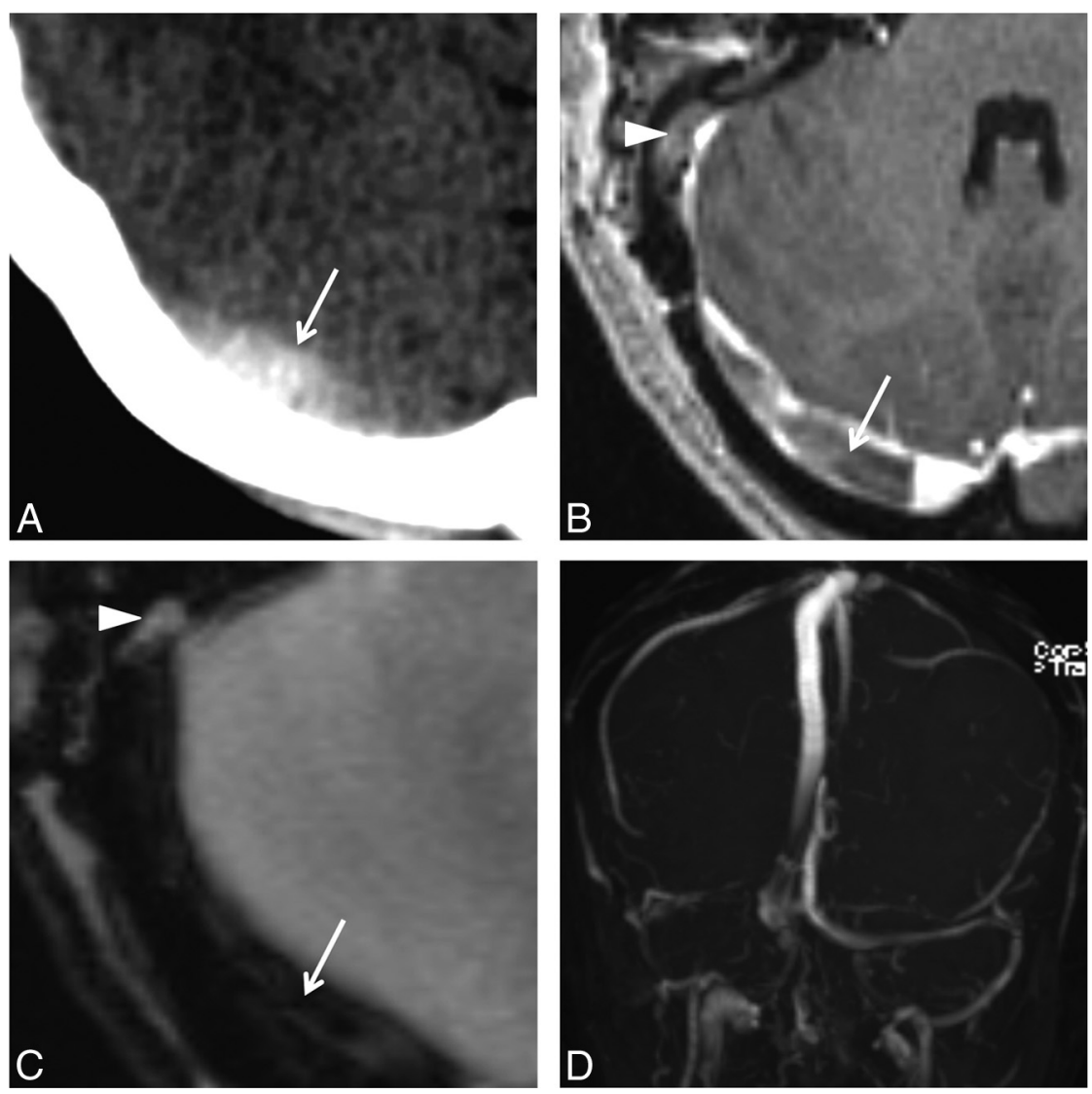

FIG 6. Transverse sinus thrombosis following SSWR. Images were obtained 11 days following surgery after the patient reported severe headaches. Noncontrast axial CT ( $A$ ) demonstrates hyperattenuation (arrow) within the right transverse sinus. On postcontrast T1-weighted MR imaging $(B)$, there is a filling defect (arrow) within the right transverse sinus, which is hypointense on the gradient-echo image (C). Note the presence of a soft-tissue graft lateral to the sigmoid sinus (arrowhead), which demonstrates no susceptibility. Maximum-intensity projection reconstruction $(D)$ demonstrates right transverse sinus thrombosis.

period but later recurred on the contralateral side: In 1 patient, this recurrence was attributed to subtle dehiscence on the other side, which was detectable on the preoperative CT scan. These cases stress the importance of careful evaluation of preoperative imaging studies for other anomalies that can cause PST, even after a sigmoid sinus wall abnormality has been identified. In a recent study, up to $70 \%$ of patients analyzed for causes of PST were found to have $>1$ vascular anomaly or variant on the symptomatic side.

\section{Limitations}

The relatively small number of patients represents a key limitation of our study. This is because postoperative imaging was only performed in those patients who presented with worrisome symptoms following surgery. In addition, a more detailed understanding of how graft materials evolve with time will require long-term follow-up imaging. Also, although the technique described is increasingly recognized as a standard approach to SSWR, variations in the use of materials across institutions may exist. It is important, therefore, for radiologists to communicate with the surgeons performing the technique to be able to interpret postoperative studies in an accurate manner.

\section{CONCLUSIONS}

Symptoms requiring postoperative imaging after SSWR include headaches, visual disturbances, and persistent/recurrent tinnitus. A variety of soft and rigid materials is used to reconstruct the sigmoid sinus wall in patients with PST arising from sigmoid sinus wall abnormalities, and these surgical materials can be easily recognized and differentiated on imaging studies. The surgical material may exert mass effect the on sinus wall, a finding that must not be mistaken for sinus thrombosis and does not require anticoagulation. Symptomatic sinus thrombosis is rare and may be distinguished from such compression by an awareness of the typical imaging appearances of the materials used in the procedure.

\section{ACKNOWLEDGMENTS}

We thank Brigitte Pocta, MLA, for assistance with revision and preparation of the manuscript.

Disclosures: Dheeraj Gandhi-UNRELATED: Royalties: Cambridge Press. Ronna Hertzano-UNRELATED: Grants/Grants Pending: National Institutes of Health,* Department of Defense, ${ }^{*}$ Comments: National Institutes of Health, transcription factors in inner ear development, RO1DC013817; Department of Defense, the molecular basis of noise induced hearing loss, MR130240. *Money paid to the institution.

\section{REFERENCES}

1. Levine SB, Snow JB Jr. Pulsatile tinnitus. Laryngoscope 1987;97:401-06 CrossRef Medline

2. Liyanage $\mathrm{SH}$, Singh A, Savundra P, et al. Pulsatile tinnitus. J Laryngol Otol 2006;120:93-97 CrossRef Medline

3. Sismanis A. Pulsatile tinnitus: a 15-year experience. Am J Otol 1998; 19:472-77 Medline

4. Sonmez G, Basekim CC, Ozturk E, et al. Imaging of pulsatile tinnitus: a review of 74 patients. Clin Imaging 2007;31:102-08 CrossRef Medline

5. Eisenman DJ. Sinus wall reconstruction for sigmoid sinus diverticulum and dehiscence: a standardized surgical procedure for a range of radiographic findings. Otol Neurotol 2011;32:1116-19 CrossRef Medline

6. Mattox DE, Hudgins P. Algorithm for evaluation of pulsatile tinnitus. Acta Otolaryngol 2008;128:427-31 CrossRef Medline

7. Dong C, Zhao PF, Yang JG, et al. Incidence of vascular anomalies and variants associated with unilateral venous pulsatile tinnitus in 242 patients based on dual-phase contrast-enhanced computed tomography. Chin Med J (Engl) 2015;128:581-85 CrossRef Medline

8. Grewal AK, Kim HY, Comstock RH 3rd, et al. Clinical presentation and imaging findings in patients with pulsatile tinnitus and sigmoid sinus diverticulum/dehiscence. Otol Neurotol 2014;35:16-21 CrossRef Medline

9. Wang GP, Zeng R, Liu ZH, et al. Clinical characteristics of pulsatile tinnitus caused by sigmoid sinus diverticulum and wall dehiscence: a study of 54 patients. Acta Otolaryngol 2014;134:7-13 CrossRef Medline 
10. Baomin L, Yongbing S, Xiangyu C. Angioplasty and stenting for intractable pulsatile tinnitus caused by dural venous sinus stenosis: a case series report. Otol Neurotol 2014;35:366-70 CrossRef Medline

11. Harvey RS, Hertzano R, Kelman SE, et al. Pulse-synchronous tinnitus and sigmoid sinus wall anomalies: descriptive epidemiology and the idiopathic intracranial hypertension patient population. Otol Neurotol 2014;35:7-15 CrossRef Medline

12. Schoeff $S$, Nicholas B, Mukherjee $S$, et al. Imaging prevalence of sigmoid sinus dehiscence among patients with and without pulsatile tinnitus. Otolaryngol Head Neck Surg 2014;150:841-46 CrossRef Medline

13. Houdart E, Chapot R, Merland JJ. Aneurysm of a dural sigmoid sinus: a novel vascular cause of pulsatile tinnitus. Ann Neurol 2000; 48:669-71 CrossRef3.3.CO\%3B2-Y Medline

14. Otto KJ, Hudgins PA, Abdelkafy W, et al. Sigmoid sinus diverticulum: a new surgical approach to the correction of pulsatile tinnitus. Otol Neurotol 2007;28:48-53 CrossRef Medline

15. Gologorsky Y, Meyer SA, Post AF, et al. Novel surgical treatment of a transverse-sigmoid sinus aneurysm presenting as pulsatile tinnitus: technical case report. Neurosurgery 2009;64:E393-94; discussion E394 CrossRef Medline

16. Keiper GL Jr, Sherman JD, Tomsick TA, et al. Dural sinus thrombosis and pseudotumor cerebri: unexpected complications of suboccipital craniotomy and translabyrinthine craniectomy. J Neurosurg 1999;91:192-97 CrossRef Medline 